\title{
An EWMA-based process mean estimator with dynamic tuning capability
}

\author{
RUEY-SHAN GUO ${ }^{1}$ and JIN-JUNG CHEN ${ }^{2}$ \\ ${ }^{1}$ Department of Industrial Management and Business Administration, National Taiwan University, Taipei, Taiwan \\ ${ }^{2}$ Department of Mechanical Engineering, National Taiwan University, Taipei, Taiwan \\ E-mail:rsguo@ccms.ntu.edu.tword3502121@ms.cc.ntu.edu.tw
}

Received April 2000 and accepted November 2001

\begin{abstract}
In this paper, we focus on the Exponentially Weighted Moving Average (EWMA) process mean estimator and its application to process adjustment. A novel dynamic-tuning EWMA estimator is proposed that has the capability of adjusting the control parameter dynamically in response to the underlying process random shifts. The current run's process mean is estimated using the EWMA equation and the newly adjusted control parameter. It is shown that the proposed estimator is very easy to implement and effective under many disturbance situations. Both industrial field data and Monte Carlo simulations are used to validate its performance.
\end{abstract}

\section{Introduction}

Manufacturing processes are subject to various special disturbances such as process drifts or shifts. From a quality control point of view, the estimation of the current process mean provides opportunities for quality monitoring and fault diagnosis. In many cases when the resulting process output deviation can be adjusted to bring the process output to the target value, a good mean estimator will certainly provide a more accurate evaluation on how much the adjustment should be made.

In this paper, we focus on the Exponentially Weighted Moving Average (EWMA) process mean estimator and its application to process adjustment. In recent years, the EWMA-based process mean estimator or adjustment has been studied by many researchers including Mac Gregor (1988), Box and Kramer (1992), Ingolfsson and Sachs (1993), Butler and Stefani (1994), Yashchin (1995), Del Castillo (1999) and also Patel and Jenkins (2000). Box and Jenkins (1976) proved that the EWMA-based mean estimation and adjustment has a Minimum Mean Square Error (MMSE) property when the underlying process disturbance follows an $\operatorname{IMA}(1,1)$ (first-order integrated moving average) process. In the semiconductor process industry, several applications of the EWMA estimator to process adjustment were proved to be effective by Sachs et al. (1995) and also Del Castillo and Hurwitz (1997) even when the underlying disturbances do not follow the IMA pattern but follow an approximately linear drift pattern (Boning et al., 1996; Smith et al., 1998).
Although effective in many applications, the EWMAbased process mean estimator still has space for enhancement. To be more specific, the estimation efficiency is highly dependent on the choice of the control parameter, and in many cases it is pre-determined for processes with small disturbances. A dynamic-tuning capability to accommodate both small and large process disturbances is required.

One such enhancement, the Adaptive Exponentially Weighted (AEW) estimator, was proposed by Yashchin (1995) for the estimation of the current mean of a process subject to abrupt changes. The AEW estimator calculates the mean based on identification of the most recent change point. Another enhancement by Patel and Jenkins (2000) used the recursive scheme for optimizing the gain of an EWMA controller under stability constraints. The objective is to minimize the asymptotic mean square error in the output with minimal a priori information. Both shift and drift disturbances are considered. In this paper, we propose a different enhancement approach. We extend the application of EWMA estimation to processes subject to random shifts of different magnitudes. A novel Dynamic-Tuning EWMA (DT-EWMA) process mean estimator is proposed. It will be shown that the proposed algorithm is very easy to implement and effective under many disturbance situations. Both industrial field data and simulations are used to validate its performance.

The rest of this paper is organized as follows. In Section 2, we introduce the DT-EWMA estimator. In Section 3, the estimation performance among the EWMA, AEW, the adaptive EWMA estimator of Patel and 
Jenkins (2000) and the DT-EWMA estimator is analyzed. In Section 4, we discuss the process adjustment applications through simulations. An industrial application of the DT-EWMA estimation is described in Section 5. Conclusions are made in Section 6.

\section{Dynamic-tuning EWMA estimator}

\subsection{Function overview}

Before introducing the proposed Dynamic-Tuning EWMA (DT-EWMA) estimator, we first review the EWMA estimation algorithm. Consider a sequence of independent observations $\left\{Y_{t}\right\}$, where $Y_{t}$ has mean $\mu_{t}$ and standard deviation $\sigma$. Then the EWMA estimation of the current process mean of observation $t$ is defined as:

$$
\begin{aligned}
\hat{\mu}_{t}= & W Y_{t}+(1-W) \hat{\mu}_{t-1}, \\
= & W Y_{t}+W(1-W) Y_{t-1}+\cdots \\
& +W(1-W)^{t-1} Y_{1}+W(1-W)^{t} Y_{0},
\end{aligned}
$$

where $W$ denotes the EWMA control parameter $(0 \leq W \leq 1)$. It has been proven that the EWMA estimator has optimality properties within the class of linear estimators for estimating the current mean of processes subject to a step change (Yashchin, 1995).

The estimation efficiency of the EWMA estimator, however, is highly dependent on the choice of the control parameter $W$. A fixed $W$ is not always efficient in mean estimation under different process disturbance conditions. An adaptive capability is required to adjust the control parameter dynamically in response to the underlying process disturbance conditions. Figure 1 shows the function diagram of the proposed DT-EWMA estimator. First the newly collected process output data is plotted using Statistical Process Control (SPC) charts. If there is any evidence of shift occurrence, the DT-EWMA estimator will trigger the dynamic-tuning capability and adjust the control parameter. The current process mean is then estimated using the newly adjusted control parameter. Notice that the SPC charts are used for two purposes; first it is used to detect if a shift has occurred; secondly it estimates how many runs ahead (when) the shift occurs.

\subsection{Dynamic-tuning algorithm}

Suppose that a sudden shift occurs at $t^{*}$; that is, $\mu_{t}=\mu_{1}$ for $t<t^{*}$ and $\mu_{t}=\mu_{2}$ for $t \geq t^{*}$. In order to swiftly compensate for the sudden shift, $W$ of the EWMA estimator in Equation (1) should be set larger instantly after the change point to capture the shift size. However, the step change occurs only once and the process mean remains unchanged after $t^{*}$. If the value of $W$ remains large, the estimator becomes oversensitive to the white noises. To overcome the trade-off between large and small $W$ and

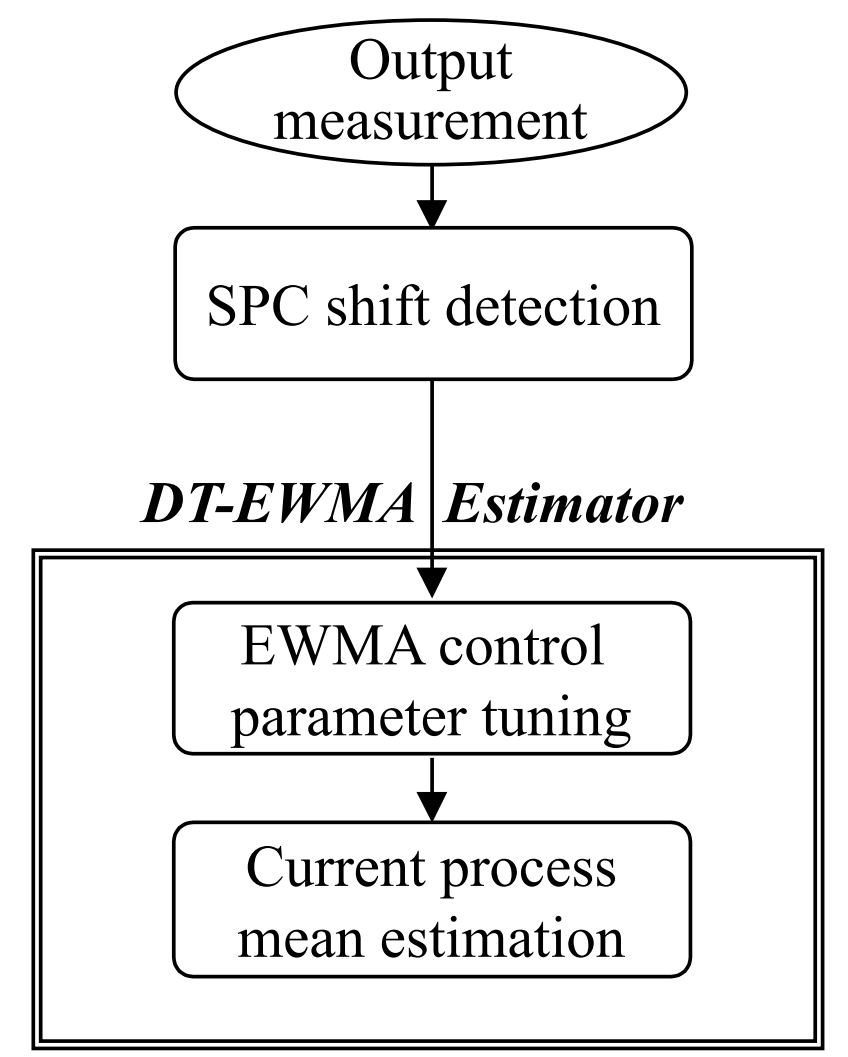

Fig. 1. Function diagram of the DT-EWMA estimator.

to design a more effective estimator for processes subject to sudden shifts, we propose an estimator with a dynamically adjusted control parameter:

$$
\hat{\mu}_{t}=W_{t} Y_{t}+\left(1-W_{t}\right) \hat{\mu}_{t-1} .
$$

In Equation (2), the values of $W_{t}$ are dynamically adjusted over time. The task now is to derive a method for adjusting $W_{t}$. Now, suppose the change point is $t^{*}$ and we do not have prior knowledge on how the process will shift away. In this case, we rely only on the sample data recently obtained from the process to estimate the shift size. In the Appendix, a theorem is provided to determine the optimal $W_{t}$ that minimizes the Mean Squared Deviation $\left(M S D_{t}\right)$ at run $t . M S D_{t}$ is defined as follows:

$$
M S D_{t}=E\left[\left(\hat{\mu}_{t}-\mu_{t}\right)^{2}\right] .
$$

The following results for $t \geq t^{*}$ are obtained:

$$
\begin{aligned}
W_{t}^{\mathrm{opt}} & =\frac{1}{t-t^{*}+1} . \\
M S D_{t}^{\mathrm{opt}} & =\frac{1}{t-t^{*}+1} \sigma^{2} .
\end{aligned}
$$

In Equation (4), $W_{t}$ decreases over time and diminishes to zero if there is no additional shift. If the change point can be detected exactly, at the detecting point $\left(t=t^{*}\right)$, the optimal initial weight will start with a full weight, i.e., a value of one. Now suppose the process is not only subject 
to shifts but also subject to other disturbances such as drifts. Therefore, a minimum value of $W_{t}$ is required to keep the estimator working for such disturbances. The resulting estimation equation becomes:

$$
\begin{aligned}
& \hat{\mu}_{t}=W Y_{t}+(1-W) \hat{\mu}_{t-1} \quad \text { for } t \geq t^{*}, \\
& W= \begin{cases}\max \left(W_{t}, W_{\mathrm{b}}\right) & \text { if dynamic-tuning loop is not } \\
& \text { completed, } \\
W_{\mathrm{b}} & \text { if dynamic-tuning loop } \\
& \text { is completed. }\end{cases}
\end{aligned}
$$

where $W_{\mathrm{b}}$ is the required minimum value of the control parameter.

In reality, the change point $t^{*}$ is unknown. As mentioned earlier, control charts are used to detect the shift occurrence in our approach. Since the signaling of a control chart only takes place some time after the actual change point, the time delay can be roughly estimated by the average run length of the control chart. Suppose now that the control chart detects the shift at the $k$ th run (Fig. 2). Then the change point can be estimated to be:

$$
\hat{t}^{*}=k-d+1,
$$

where $d$ is control chart run length. Equations (4) and (6) can be then applied. The complete algorithm of the DTEWMA estimator is illustrated in Fig. 3.

From Equations (4) and (7), the control parameter of the DT-EWMA estimator has a starting value at the detection point:

$$
W_{k}=\frac{1}{k-\hat{t}^{*}+1}=\frac{1}{d} .
$$

That is, the estimator will take into consideration $d$ data points prior to the detection point $k$ while estimating $\mu_{t}$. Notice that the starting weight value is $1 / d$, instead of one, to take into account the data points between the detection point and the change point to provide a more accurate estimation of current mean. The control parameter continues to diminish over time until it reaches $W_{\mathrm{b}}$ and is fixed at that minimum required level afterwards. This control cycle holds on until the control chart signals again.

Notice that the values of $d$ depend on the design of the control chart. Various Average Run Lengths (ARLs) for different shift sizes are reported in the literature by Champ and Woodhall (1987), Crowder (1987) and also Lucas and Saccucci (1990). In this paper, two EWMA control charts were used for the shift detection in the

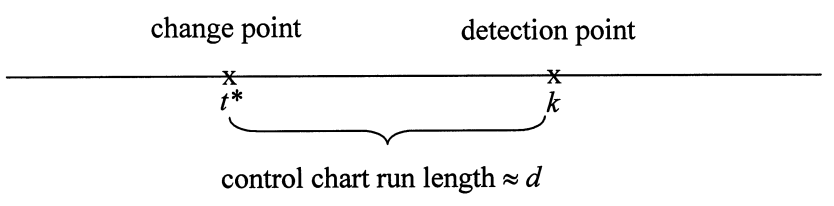

Fig. 2. The run length for the control chart to detect a process shift. following simulation case study. Their design parameters and $A R L \mathrm{~s}$ which were estimated based on the Markovian approximation (Lucas and Saccucci, 1990.) are shown in Table 1. For EWMA_1 control chart, $d$ is roughly equal to two at the detection of a shift size of 3 $\sigma$. For EWMA_2 control chart, $d$ is roughly equal to four at the detection of a shift size of $2 \sigma$. Figure 4 shows the potential changes of the control parameter over time under these control chart designs.

\section{Process mean estimation performance analysis}

In this section, we analyze the estimation performance among the EWMA, AEW (Yaschin, 1995), the adaptive EWMA of Patel and Jenkins (2000) and the DT-EWMA estimator using simulations. In these simulations, we consider a process with mean $\mu_{t}=\mu_{1}$ and standard deviation $\sigma$ for $t<t^{*}$, and $\mu_{t}=\mu_{1}+s \sigma$ for $t \geq t^{*}$ (i.e., a sudden shift occurs at $t^{*}$ ). The estimation performance is then characterized by a performance index, normalized Mean Square Estimation Error (MSEE). The smaller the value, the better the estimation performance. The normalized mean square estimation error is denoted as $M S E E / \sigma^{2}$ and defined as:

$$
M S E E / \sigma^{2}=\left\{\frac{1}{n} \sum_{j=1}^{n}\left(\hat{\mu}_{t^{*}+j-1}-\mu_{t^{*}+j-1}\right)^{2}\right\} / \sigma^{2} .
$$

Here the $M S E E / \sigma^{2}$ index is calculated based on the simulation results of 100 runs and 2000 simulation cycles. For each simulation cycle, the shift occurs at the 51st run so $t^{*}=51$ and $n=50$.

Figure 5 shows the results of the simulations (MSEE $/ \sigma^{2}$ versus $s$ ). Notice that we have chosen each method's parameters ( $W_{\mathrm{b}}$ for DT-EWMA, $r$ and $h$ for AEW, $\delta$ and $\varepsilon$ for Patel and Jenkins) so that they all have roughly the same $M S E E / \sigma^{2}$ when the shift size is zero. As can be seen, the adaptive EWMA estimator of Patel and Jenkins performs the worst under different shift sizes. The other three estimators perform roughly the same when the magnitude of the shift is smaller than $2 \sigma$. However, both the AEW and DT-EWMA estimators perform better than the EWMA estimator when the magnitude of the shift is larger than $2 \sigma$. That is, both the AEW and DTEWMA estimators have a more stable performance under different magnitudes of the shifts. This is due to the fact that the AEW estimator has an "adaptive capability" while the DT-EWMA estimator has a "dynamic-tuning capability" once a shift occurs. The adaptive algorithm in the work of Patel and Jenkins (2000) is a very conservative approach in updating the control parameter once a shift occurs. Its adaptive performance is not very effective.

During the simulations, we also found that the algorithm for estimating the change point in the AEW 


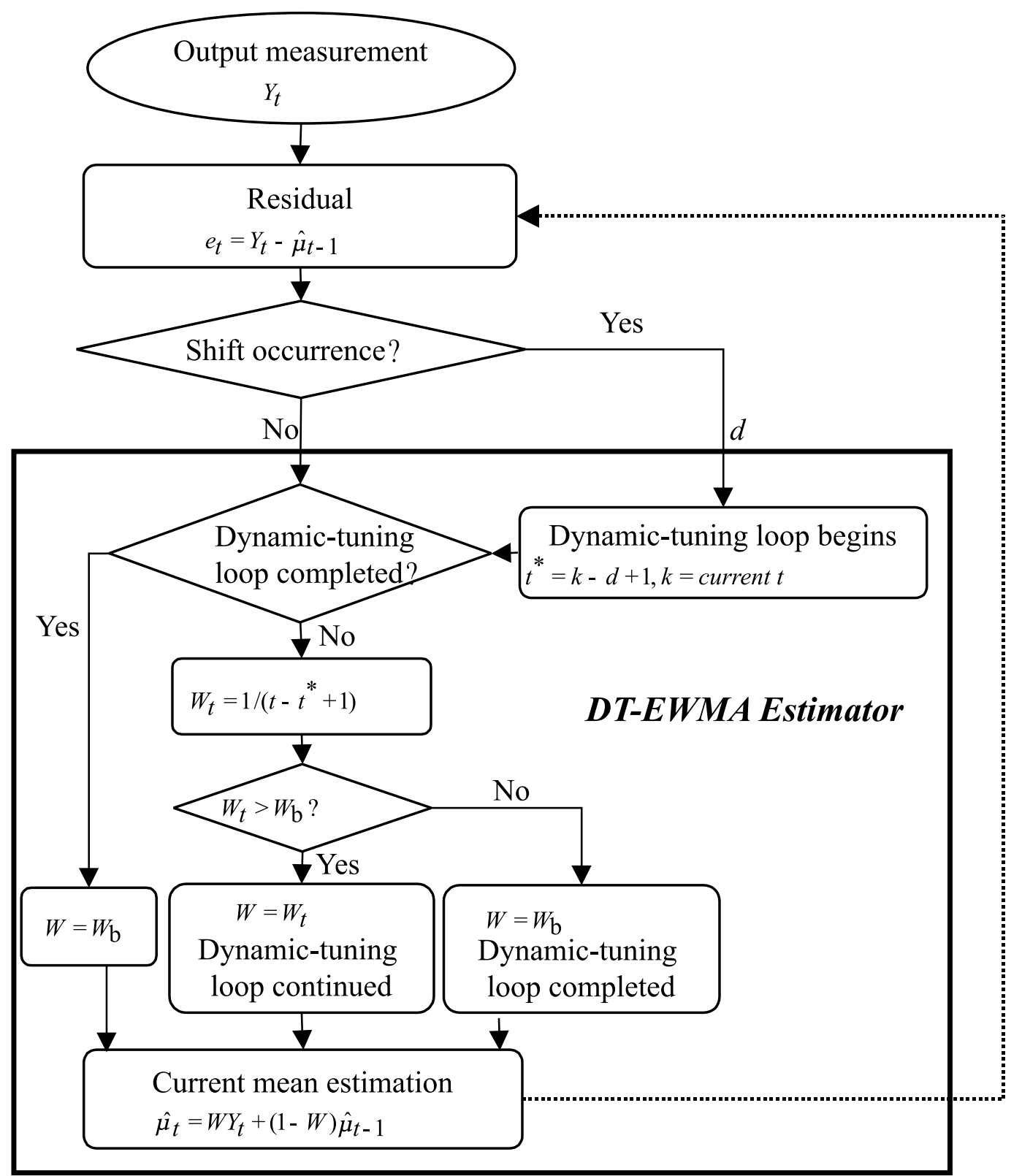

Fig. 3. The complete algorithm of the DT-EWMA estimator.

estimator requires an intensive computational effort. In the DT-EWMA estimator, the location of the change point is

Table 1. Average run length for two EWMA control charts

\begin{tabular}{|c|c|c|c|}
\hline Shift size & $A R L$ & $\begin{array}{l}E W M A_{-} 1 \\
W_{l}=0.6 \\
C L=3.285 \sigma_{l}\end{array}$ & $\begin{array}{l}E W M A \_2 \\
W_{m}=0 . \overline{3} 3 \\
C L=3.25 \sigma_{m}\end{array}$ \\
\hline $0 \sigma$ & $A R L_{0}$ & 1003.44 & 1007.44 \\
\hline $1 \sigma$ & $A R L_{1}$ & 31.55 & 15.87 \\
\hline $2 \sigma$ & $A R L_{1}$ & 4.55 & 3.90 \\
\hline $3 \sigma$ & $A R L_{1}$ & 2.06 & 2.27 \\
\hline $4 \sigma$ & $A R L_{1}$ & 1.36 & 1.67 \\
\hline
\end{tabular}

estimated by the $A R L$ of the adopted control charts. Although the search of the change point by the AEW method might be more rigorous than by the DT-EWMA estimator, the performance is roughly the same as indicated in Fig. 5. For the computational complexity comparison, suppose the current process run is the $i$ th run. In the worst case when there is no shift occurrence till the $i$ th run, we have estimated that the required number of computations by the AEW method at the $i$ th run is roughly equal to $i(i-1) / 2$. In the DT-EWMA estimator, if two control charts are used for shift detection, the number of computations is only two at each run. Suppose there is no shift until run 200 , the total number of calculations from run 1 to run 200 by the AEW method will 


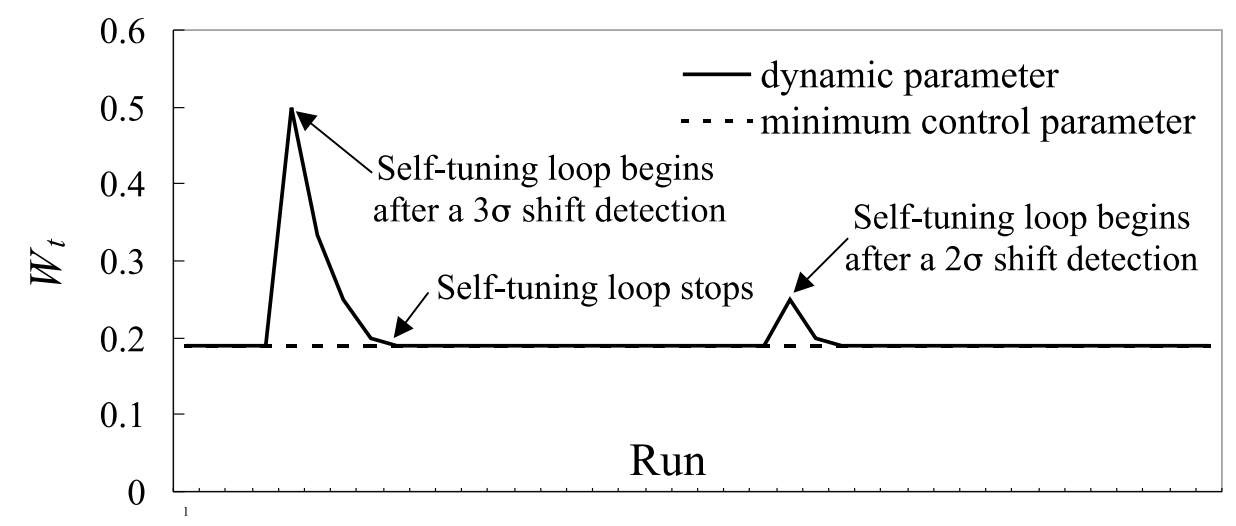

Fig. 4. Dynamic and minimum control parameters.

be 1333 300. The number of calculations using the DTEWMA method is only 400. There is a significant difference. Figure 6 also shows the results for DT-EWMA estimator under different shift magnitudes and $W_{\mathrm{b}}$. As can be seen, the performance is not very sensitive to the selection of $W_{\mathrm{b}}$.

\section{Process adjustment simulations}

\subsection{Process model and disturbance}

In many manufacturing processes, when the process output deviation can be adjusted to bring the process output to the target value, the process mean estimator also provides information on the magnitude of the required adjustment. In this section, we conduct Monte Carlo simulations to study the adjustment performance based on the EWMA and DT-EWMA estimators.

First we need to define the process model and process disturbance. Suppose the underlying process model and disturbance can be approximated as:

$$
Y_{t}=\alpha+\beta X_{t}+\varepsilon_{t}+\delta_{t} .
$$

$$
\begin{aligned}
\varepsilon_{t} & \sim N\left(0, \sigma^{2}\right) . \\
\delta_{t} & =c \sigma t+\tau_{t} .
\end{aligned}
$$

Here $\alpha$ is the process constant, $\beta$ is the process gain, $X$ is the input setting, $\varepsilon$ is the white noise and $\delta$ is the special disturbance. Notice that in Equation (12) two types of disturbance are simulated: process drift and shift. The process drift follows a linear drift pattern and deviates from the target $(T)$ at the speed of $c \sigma$. The shift is modeled as a random shift with the following pattern:

$$
\tau_{t}= \begin{cases}\tau_{t-1} & \text { with probability } 1-p \\ \tau_{t-1}+S & \text { with probability } p\end{cases}
$$

where $p$ is the probability of the shift occurrence. The shift magnitude $S$ is a random variable and $S \sim N\left(\mu_{S}, \sigma^{2}\right)$.

In the Monte Carlo simulations, the values of the above parameters are chosen as: $\alpha=3000, \beta=10$, $\sigma=50, c=0.02$, and $T=3500$. As for the random shift, it is simulated in four cases and the occurrence probability $(p)$ and magnitude $(S)$ are summarized in Table 2. For example, in the first case the random shift has a low probability of occurrence and a smaller shift magnitude.

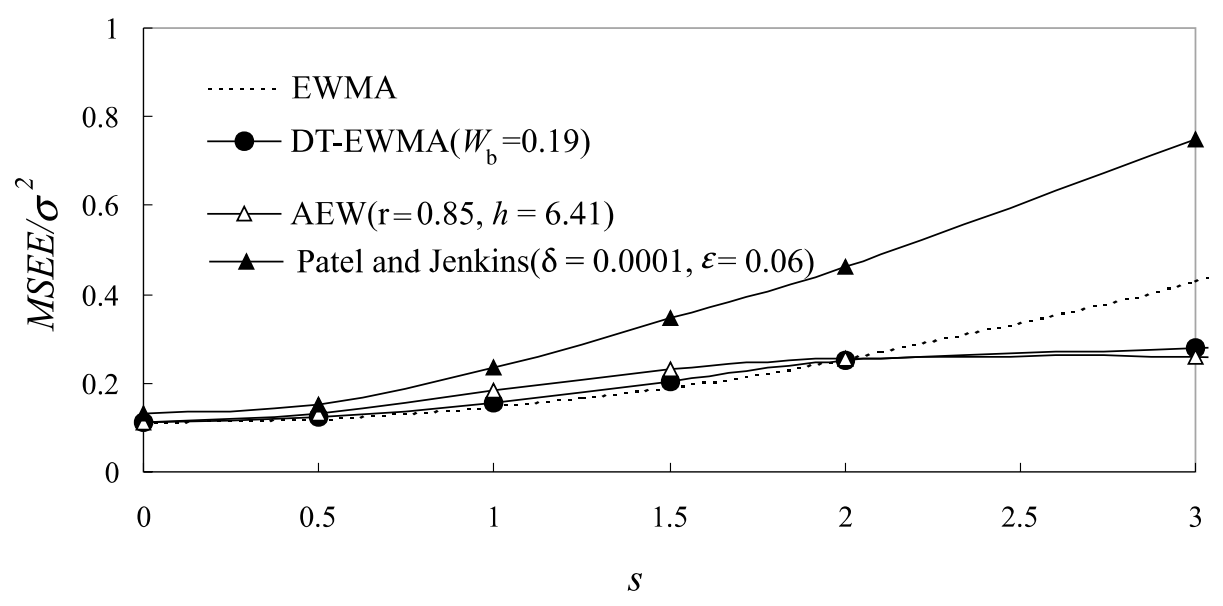

Fig. 5. Process mean estimation performance of four estimators under different shift magnitudes. 


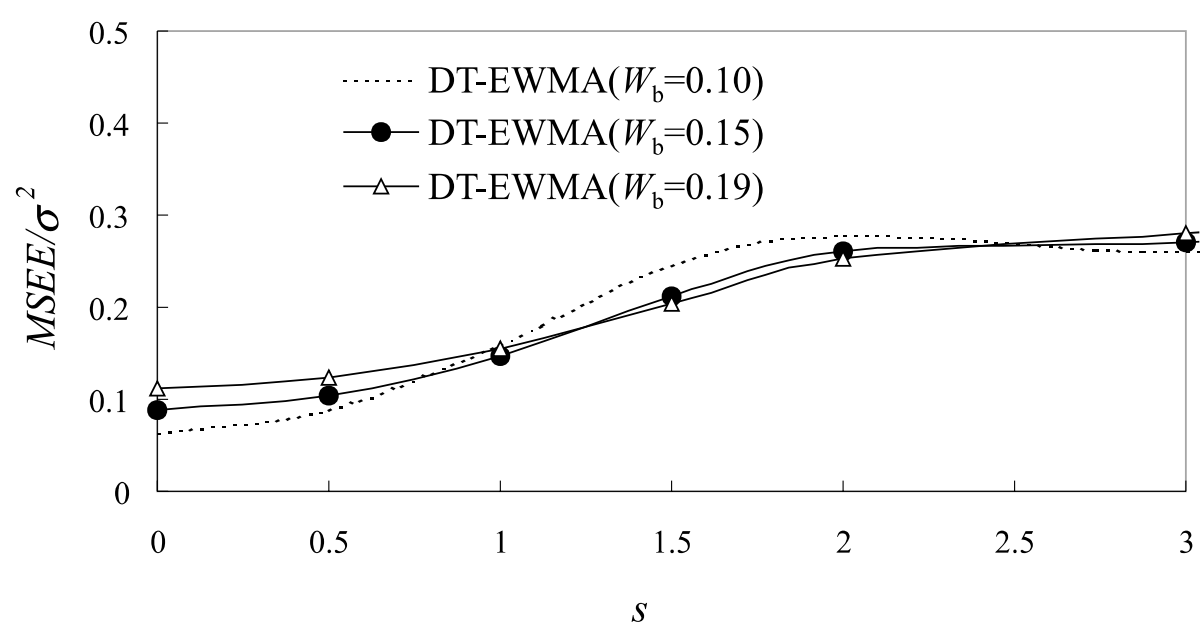

Fig. 6. Process mean estimation performance of the DT-EWMA estimator under different shift magnitudes and $W_{\mathrm{b}}$.

In the fourth case, the random shift has a high probability of occurrence and a larger shift magnitude.

In the process adjustment simulations, once the current run's mean is estimated, the adjustment for the next run is achieved by tuning the input setting as:

$$
X_{t+1}-X_{t}=\frac{T-\hat{\mu}_{t}}{\beta} .
$$

As for the adjustment performance characterization, we use the normalized mean square error as the performance index. The smaller the value, the better the performance. The normalized mean square error is denoted as $M S E / \sigma^{2}$ and is defined as:

$$
M S E / \sigma^{2}=\left\{\frac{1}{n} \sum_{t=1}^{n}\left(Y_{t}-T\right)^{2}\right\} / \sigma^{2} .
$$

Here the $M S E / \sigma^{2}$ index is calculated based on the simulation results of 2000 runs and 200 simulation cycles.

\subsection{Monte Carlo simulation results}

Simulation results for Case 3 and Case 4 are shown in Figs. 7 and 8. Figure 9 shows the results of the average of Case 1 to Case 4 and represents the situation when the disturbance information is unknown and all types of disturbance are possible.

Based on these results, we have the following conclusions:

- When there are only small process drifts or small shifts, as happened in Cases 1 and 3, the process adjustment based on the EWMA estimator is very effective in compensating for these small disturbances. As a result, the DT-EWMA estimator has a similar adjustment performance as the EWMA estimator.

- When there are large process shifts, as happened in Cases 2 and 4, the adjustment performance based on the DT-EWMA estimator is much better than that of the EWMA estimator with fixed control parameter. The performance of the DT-EWMA estimator is not very dependent on the minimum control parameter.

- The DT-EWMA estimator is very effective at compensating for disturbances of random shifts and linear drifts.

\section{CMP process case study}

In this section, we apply both EWMA and DT-EWMA estimators to an industrial process and compare their estimation performance. The selected industrial process, Chemical-Mechanical Polishing (CMP) process (Fig. 10), is considered to be a strategically important technology for IC fabrication. Because the process includes mechanical abrasion of the surface, the polishing pad wears rapidly, which results in a decreasing and irregular runto-run process removal rate.

A simple strategy for controlling the CMP process is to estimate the run-to-run process removal rate and then adjust the polishing time based on the estimated removal rate (Boning et al., 1996). The estimation, however, is not

Table 2. Four cases modelled in our simulations

\begin{tabular}{lllll}
\hline & Case 1 & Case 2 & Case 3 & Case 4 \\
\hline$P$ & 0.005 & 0.005 & 0.05 & 0.05 \\
$S$ & $N\left(0, \sigma^{2}\right)$ & $N\left(3 \sigma, \sigma^{2}\right)$ & $N\left(0, \sigma^{2}\right)$ & $N\left(3 \sigma, \sigma^{2}\right)$ \\
\hline
\end{tabular}




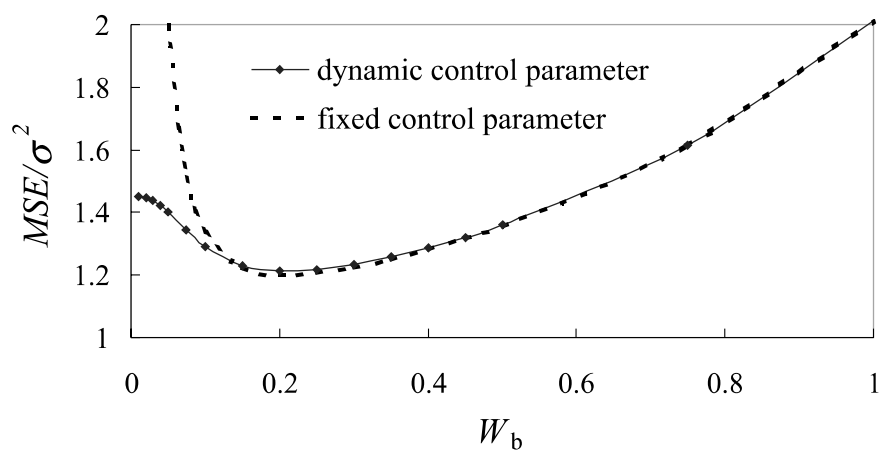

Fig. 7. Simulation results for case 3 .

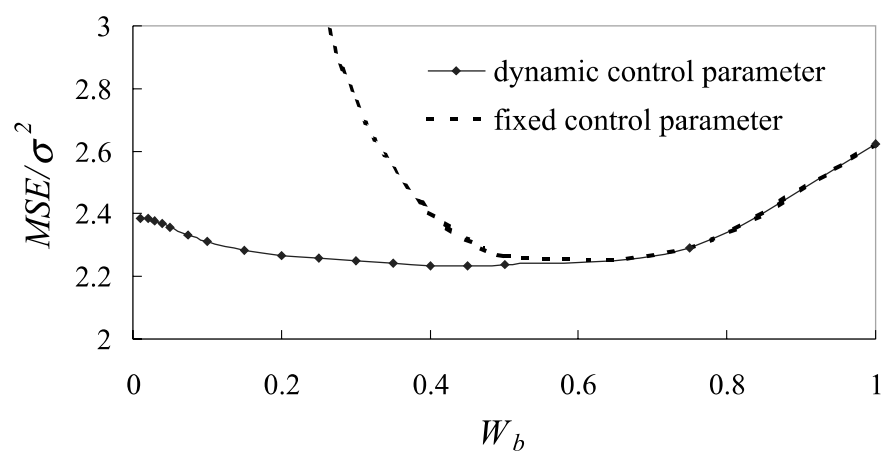

Fig. 8. Simulation results for case 4 .

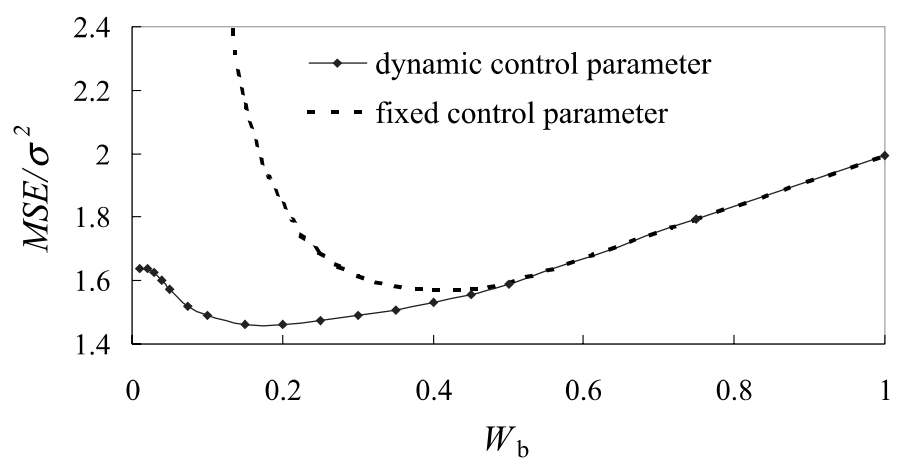

Fig. 9. Average results of cases 1, 2, 3, and 4 .

easy for a field process engineer. Figure 11 shows data on the removal rate sampled from a local wafer fab. It can be observed that there are several shifts of different magnitudes (because of the replacement of the pads) and a decreasing trend of removal rate (because of the wear-out of the pads). The pattern of the field data is actually more complicated than the process model we assume in the previous sections. Nevertheless, they are still valuable to our study.

Since the replacement of the pad usually results in a shift, we have used this prior information in our case study. In applying the DT-EWMA and EWMA to the

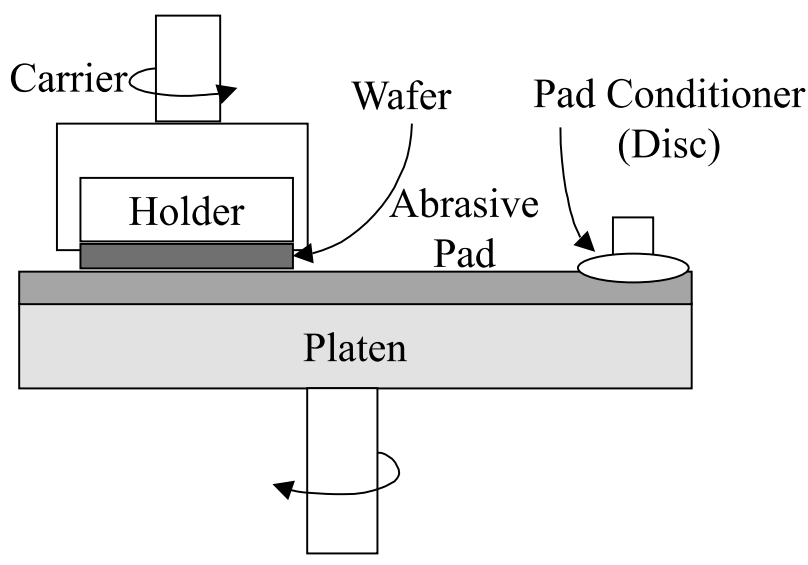

Fig. 10. Schematic of the CMP process equipment.

CMP data, we reset the estimation right after the pad replacement so that the estimation can be more accurate.

Figure 12 shows the results of $M S E E$ versus $W_{\mathrm{b}}$ for EWMA and DT-EWMA estimators. The fact that the minimum MSEE occurs at around $W_{\mathrm{b}}=0.8$ indicates that the underlying process mean is changing very frequently and a higher value of $W$ is required. Although both the EWMA and DT-EWMA estimators have the same estimation performance at around $W_{\mathrm{b}}=0.8$, the DT-EWMA estimator is less sensitive to the selection of $W_{\mathrm{b}}$. In real practice, the value of the control parameter is usually selected to be less than 0.5 . As a result, such a practice will certainly fail using the conventional EWMA estimator. On the other hand, the DT-EWMA estimator appears to be quite robust and the selection of $W_{\mathrm{b}}$, even for values less than 0.5, will not cause a significant increase in MSEE.

\section{Conclusions}

In this paper, we present an EWMA-based mean estimator with dynamic-tuning capability for processes subject to random shifts of different magnitudes. The DT-EWMA estimator uses the criteria of minimum mean square deviation to derive the optimal control parameter in response to the random shift disturbances. Through the simulation and case study, we conclude that:

- The DT-EWMA estimator, similar to the AEW estimator, has a more stable estimation performance under different magnitudes of random shifts than the other approaches. A possible extension of this approach could be the determination of the change point by a more rigorous method instead of the control chart and the $A R L$ approach if the computation effort is not an issue.

- The dynamic-tuning capability of the DT-EWMA estimator is analytically derived and is very easy to implement.

- The process adjustment that is based on the DTEWMA estimator is very effective for processes 


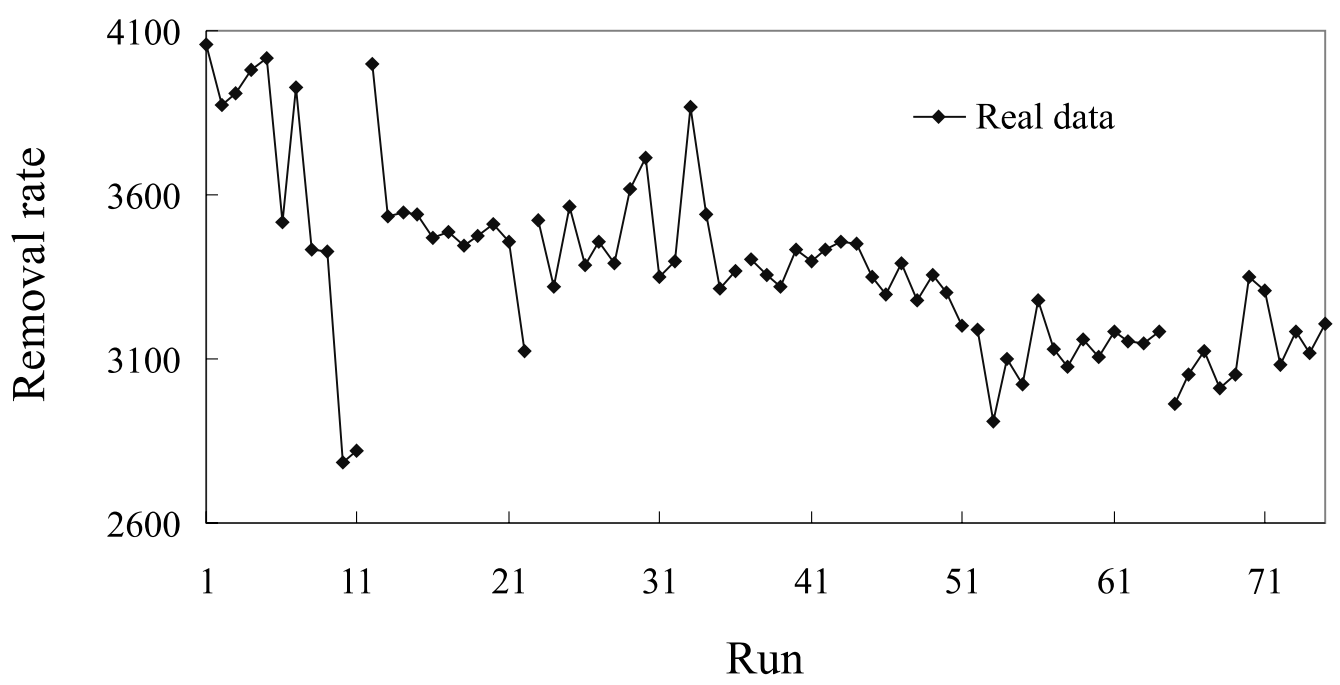

Fig. 11. Removal rate data of the CMP process.

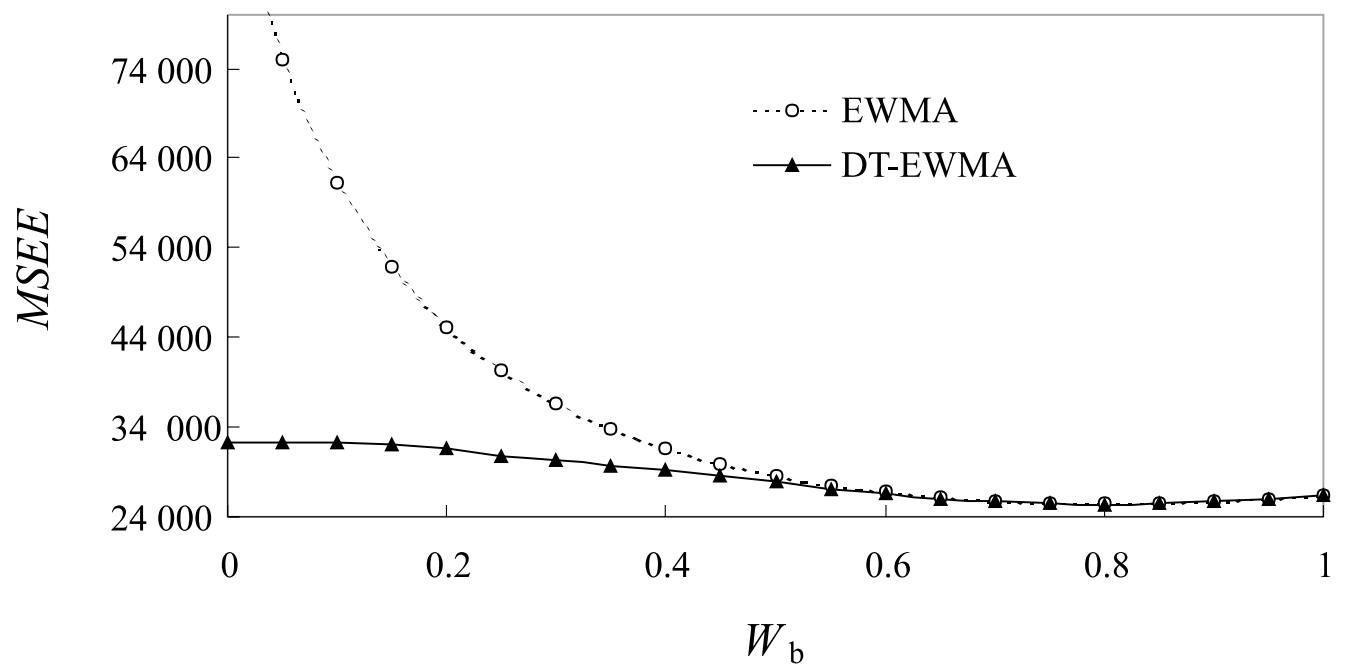

Fig. 12. A comparison of the performances of the EWMA and DT-EWMA estimators.

subject to different magnitudes of random shifts and linear drifts.

\section{Acknowledgement}

The authors would like to thank Dr. Argon Chen of the National Taiwan University for his valuable comments during the preparation of this paper. The work was supported by the National Science Council of the Republic of China under Grant NSC88-2416-H-002-055 and NSC89-2416-H-002-044.

\section{References}

Boning, D., Moyne, W., Smith, T., Moyne, J., Hurwitz, A., Shellman, S., Moyne, J., Trefeyan, R. and Taylor, J. (1996) Run by run process control of chemical - mechanical polishing. IEEE Transactions on Components, Packaging, and Manufacturing Technology - Part C, 19(4), 307-313.

Box, G. and Jenkins, M. (1976) Time Series Analysis - Forecasting and Control, Holden-Day, San Francisco, CA.

Box, G. and Kramer, T. (1992) Statistical process monitoring and feedback adjustment - a discussion. Technometrics, 34(3), 251-267.

Butler, S. and Stefani, J. (1994) Supervisory run-to-run control of polysilicon gate etch using in situ ellipsometry. IEEE Transactions on Semiconductor Manufacturing, 7(2), 193-201.

Champ, C.W. and Woodall, W.H. (1987) Exact result for Shewhart control charts with supplementary runs rules. Technometrics, 29(4), 393-399.

Crowder, S.V. (1987) A simple method for studying run-length distributions of exponentially weighted moving average charts. Technometrics, 29(4), 401-407.

Del Castillo, E. (1999) Long run and transient analysis of a double EWMA feedback controller. IIE Transactions, 31(12), 1157-1169.

Del Castillo, E. and Hurwitz, A.N. (1997) Run-to-run process control: literature review and extensions. Journal of Quality Technology, 29(2), 184-186. 
Ingolfsson, A. and Sachs, E. (1993) Stability and sensitivity of an EWMA controller. Journal of Quality Technology, 25(4), 271-287.

Lucas, J.M. and Saccucci, M.S. (1990) Exponentially weighted moving average control schemes: properties and enhancements. Technometrics, 32(1), 1-29.

MacGregor, J.F. (1988) On line statistical process control. Chemical Engineering Progress, 84, 21-31.

Patel, N.S. and Jenkins, S.T. (2000) Adaptive optimization of run-torun controllers: the EWMA example. IEEE Transactions on Semiconductor Manufacturing, 13(1), 97-107.

Sachs, E., Hu, A. and Ingolfsson, A. (1995) Run by run process control: combining SPC and feedback control. IEEE Transactions on Semiconductor Manufacturing, 8(1), 26-43.

Smith, T., Boning, D., Stefani, J. and Butler, S. (1998) Run by run advanced process control of metal sputter deposition. IEEE Transactions on Semiconductor Manufacturing, 11(2), 276-284.

Yashchin, E. (1995) Estimating the current mean of a process subject to abrupt changes. Technometrics, 37(3), 311-323.

\section{Appendix}

Theorem 1. Given a process model, suppose that $\mu_{t}$ changes once somewhere between time $t^{*}-1$ and $t^{*}$; that is, $\mu_{t}=\mu_{1}$ for $t<t^{*}$ and $\mu_{t}=\mu_{2}$ for $t \geq t^{*}$ but we do not have any prior information on the size of the $\mu_{2}$. Then the EWMA estimator with a dynamically adjusted control parameter in Equation (2) minimizes $M S D_{t}$ if

$$
W_{t}=\frac{1}{t-t^{*}+1},
$$

for all $t \geq t^{*}$ and the observation at the time $t^{*}$ is known.

Proof. Given the weight

$$
W_{t}=\frac{1}{t-t^{*}+1},
$$

for any $t^{*} \leq t<\tau$,

$$
\begin{aligned}
\hat{\mu}_{\tau} & =W_{\tau} Y_{\tau}+\left(1-W_{\tau}\right) \hat{\mu}_{\tau-1}, \\
& =W_{\tau} Y_{\tau}+\left(1-W_{\tau}\right)\left[W_{\tau} Y_{\tau-1}+\left(1-W_{\tau}\right) \hat{\mu}_{\tau-2}\right] .
\end{aligned}
$$

Substituting

$$
W_{\tau-1}=\frac{1}{\tau-t^{*}}
$$

into the above equation, we obtain

$$
\begin{aligned}
\hat{\mu}_{\tau}= & W_{\tau}\left(\mu_{2}+\varepsilon_{\tau}\right)+\left(1-W_{\tau}\right)\left[\frac{1}{\tau-t^{*}}\left(\mu_{2}+\varepsilon_{\tau-1}\right)+\frac{\tau-t^{*}-1}{\tau-t^{*}} \hat{\mu}_{\tau-2}\right], \\
= & W_{\tau}\left(\mu_{2}+\varepsilon_{\tau}\right)+\left(1-W_{\tau}\right) \\
& \times\left\{\frac{1}{\tau-t^{*}}\left(\mu_{2}+\varepsilon_{\tau-1}\right)+\frac{\tau-t^{*}-1}{\tau-t^{*}}\left[W_{\tau-2} Y_{\tau-2}+\left(1-W_{\tau-2}\right) \hat{\mu}_{\tau-3}\right]\right\} .
\end{aligned}
$$

Again, substituting

$$
W_{\tau-2}=\frac{1}{\tau-t^{*}-1},
$$

into the above equation, we obtain

$$
\begin{aligned}
\hat{\mu}_{\tau}= & W_{\tau}\left(\mu_{2}+\varepsilon_{\tau}\right)+\left(1-W_{\tau}\right) \\
& \times\left\{\frac{1}{\tau-t^{*}}\left(\mu_{2}+\varepsilon_{\tau-1}\right)+\frac{\tau-t^{*}-1}{\tau-t^{*}}\right. \\
& \left.\times\left[\frac{1}{\tau-t^{*}-1}\left(\mu_{2}+\varepsilon_{\tau-2}\right)+\frac{\tau-t^{*}-2}{\tau-t^{*}-1} \hat{\mu}_{\tau-3}\right]\right\} . \\
= & W_{\tau}\left(\mu_{2}+\varepsilon_{\tau}\right)+\left(1-W_{\tau}\right)\left\{\frac{1}{\tau-t^{*}}\left(\mu_{2}+\varepsilon_{\tau-1}\right)\right. \\
& +\frac{\tau-t^{*}-1}{\tau-t^{*}}\left[\frac{1}{\tau-t^{*}-1}\left(\mu_{2}+\varepsilon_{\tau-2}\right)\right. \\
& \left.\left.+\frac{\tau-t^{*}-2}{\tau-t^{*}-1}\left(W_{\tau-3} Y_{\tau-3}+\left(1-W_{\tau-3}\right) \hat{\mu}_{\tau-4}\right)\right]\right\} . \\
= & W_{\tau}\left(\mu_{2}+\varepsilon_{\tau}\right)+\left(1-W_{\tau}\right)\left\{\frac{2}{\tau-t^{*}}\left(\mu_{2}+\varepsilon_{\tau-1}\right)+W_{\tau} \varepsilon_{\tau}\right. \\
& +\left(1-W_{\tau}\right) \frac{1}{\tau-t^{*}} \sum_{i=\tau-2}^{\tau-1} \varepsilon_{i}+\frac{\tau-t^{*}-2}{\tau-t^{*}-1}\left(W_{\tau-3} Y_{\tau-3}\right. \\
& \left.\left.\left.+\left(1-W_{\tau-3}\right) \hat{\mu}_{\tau-4}\right)\right]\right\} .
\end{aligned}
$$

By recursively substituting

$$
W_{\tau-3}=\frac{1}{\tau-t^{*}-2}, \ldots, W_{t^{*}}=1,
$$

into the equation, we finally have

$$
\hat{\mu}_{\tau}=\mu_{2}+W_{\tau} \varepsilon_{\tau}+\left(1-W_{\tau}\right) \frac{1}{\tau-t^{*}} \sum_{i=t^{*}}^{\tau-1} \varepsilon_{i} .
$$

The $M S D$ at $\tau$ is:

$$
\begin{aligned}
M S D_{\tau} & =E\left[\left(\hat{\mu}_{\tau}-\mu_{\tau}\right)^{2}\right], \\
& =E\left[\left(W_{\tau} \varepsilon_{\tau}+\left(1-W_{\tau}\right) \frac{1}{\tau-t^{*}} \sum_{i=t^{*}}^{\tau-1} \varepsilon_{i}\right)^{2}\right], \\
& =W_{\tau}^{2} \sigma^{2}+\left(1-W_{\tau}\right)^{2} \frac{1}{\tau-t^{*}} \sigma^{2} .
\end{aligned}
$$

By minimizing $M S D_{\tau}$ with respect to $W_{\tau}$, we obtain the optimal control parameter at $\tau$.

$$
W_{\tau}^{\mathrm{opt}}=\frac{1}{\tau-t^{*}+1}
$$

and the optimal $M S D$

$$
M S D_{\tau}^{\mathrm{opt}}=\frac{1}{\tau-t^{*}+1} \sigma^{2}
$$

We have shown that given

$$
\begin{gathered}
W_{t}=\frac{1}{t-t^{*}+1} \text { for } t^{*} \leq t<\tau, \\
W_{\tau}^{\mathrm{opt}}=\frac{1}{\tau-t^{*}+1} .
\end{gathered}
$$

That is, if 


$$
W_{t^{*}}^{\mathrm{opt}}=\frac{1}{t^{*}-t^{*}+1}=1,
$$

then recursively we can obtain

$$
W_{t}^{\mathrm{opt}}=\frac{1}{t-t^{*}+1}
$$

for $\forall t \geq t^{*}$.

Now, we show that $W_{t^{*}}^{\text {opt }}=1$. Because

$$
\begin{aligned}
\hat{\mu}_{t^{*}} & =W_{t^{*}} Y_{t^{*}}+\left(1-W_{t^{*}}\right) \hat{\mu}_{t^{*}-1}, \\
& =Y_{t^{*}}+\left(1-W_{t^{*}}\right)\left(\hat{\mu}_{t^{*}-1}-Y_{t^{*}}\right) .
\end{aligned}
$$

Given the output at time $t^{*}$ is observed to be $y_{t^{*}}$

$$
y_{t^{*}}=\mu_{t^{*}}+\varepsilon_{t^{*}}
$$

the $M S D$ at $t^{*}$ is:

$$
\begin{aligned}
M S D_{t^{*}}= & E\left[\left(\hat{\mu}_{t^{*}}-\mu_{t^{*}}\right)^{2} \mid Y_{t^{*}}=y_{t^{*}}\right], \\
= & E\left\{\left[Y_{t^{*}}+\left(1-W_{t^{*}}\right)\left(\hat{\mu}_{t^{*}-1}-Y_{t^{*}}\right)\right.\right. \\
& \left.\left.-\left(y_{t^{*}}-\varepsilon_{t^{*}}\right)\right]^{2} \mid Y_{t^{*}}=y_{t^{*}}\right\}, \\
= & \left.E\left\{\left[\left(1-W_{t^{*}}\right)\left(\hat{\mu}_{t^{*}-1}-Y_{t^{*}}\right)+\varepsilon_{t^{*}}\right)\right]^{2}\right\}, \\
= & \left(1-W_{t^{*}}\right)^{2}\left(\hat{\mu}_{t^{*}-1}-y_{t^{*}}\right)^{2}+\sigma^{2}
\end{aligned}
$$

Minimizing $M S D_{t^{*}}$ with respect to $W_{t^{*}}$, we obtain optimal weight $W_{t^{*}}^{\mathrm{opt}}=1$ and $M S D_{t^{*}}^{\mathrm{opt}}=\sigma^{2}$.

Therefore,

$$
W_{t}^{\mathrm{opt}}=\frac{1}{t-t^{*}+1}
$$

and

$$
M S D_{t+1}^{\mathrm{opt}}=\frac{1}{t-t^{*}+1} \sigma^{2} \text { for } \forall t \geq t^{*}
$$

\section{Biographies}

Ruey-Shan Guo received his Ph.D. degree in Mechanical Engineering from MIT in 1991 and an MBA degree from San Jose State University in 1994. From 1991 to 1995, he was a Senior Process Engineer at National Semiconductor Fairchild Research Center, Santa Clara, CA. From 1995 till now, he has been an Associate Professor in the Graduate Institute of Business Administration and Graduate Institute of Industrial Engineering of National Taiwan University. During his current position, he has been a principal investigator and consultant to many industry- and government-funded projects. His current research interests include advanced quality control, run-to-run process control, CIM, and supply chain management. He won the excellent teaching award from National Taiwan University twice and the best annual journal paper from the Chinese Management Academics Association. Dr. Guo is a member of IEEE.

Jin-Jung Chen received a Ph.D. degree in Mechanical Engineering from the National Taiwan University in 2000. He is currently a manager in the Product Division of the Mustang Industrial Corporation in Taiwan.

Contributed by the Design of Experiments and Robust Designs Department 